\title{
Psicología y Destrucción del Psiquismo: La Utilización Profesional del Conocimiento Psicológico para la Tortura de Presos Políticos
}

\author{
David Pavón-Cuéllar \\ Universidad Michoacana de San Nicolás de Hidalgo, México.
}

\begin{abstract}
Resumen: El artículo aborda el tema de la utilización de la Psicología para la tortura de presos políticos en el mundo y especialmente en América Latina. Primero se incursiona en el reciente debate sobre los psicólogos torturadores de los Estados Unidos. Luego se recuerdan los precedentes del empleo de la Psicología para torturar en la Alemania nazi, la España franquista, la represión colonial francesa en Argelia y la estrategia militar estadounidense durante la Guerra Fría. La consideración de tales precedentes y del reciente debate en los Estados Unidos permite llegar a una representación general de la forma en que la Psicología opera en la tortura como forma de supresión y desintegración del psiquismo. Esta representación general guía un análisis de los casos de cuatro profesionales de la salud mental que pusieron sus profesiones al servicio de regímenes autoritarios latinoamericanos para torturar a presos políticos entre los años $60 \mathrm{y}$ 70 del siglo XX: el psiquiatra mexicano Salvador Roquet, el psicoanalista brasileño Amílcar Lobo Moreira, el psicólogo uruguayo Dolcey Brito y el psicólogo chileno Hernán Tuane.
\end{abstract}

Palabras clave: Dictadura, Tortura, Psicología, Psicoanálisis, Psiquiatría.

\section{Psicologia e Destruição do Psiquismo: a Utilização Profissional do Conhecimento Psicológico para a Tortura de Presos Políticos}

Resumo: O artigo aborda o tema da utilização da Psicologia para a tortura de presos políticos no mundo e especialmente na América Latina. Primeiro discorre-se acerca do recente debate sobre os psicólogos torturadores dos Estados Unidos. Logo recorda-se os precedentes do emprego da Psicologia para torturar na Alemanha nazista, na Espanha franquista, na repressão colonial francesa na Argélia e na estratégia militar estadunidense durante a Guerra Fria. A consideração de tais precedentes e do recente debate nos Estados Unidos permite chegar a uma representação geral da forma com que a Psicologia opera na tortura como forma de supressão e desintegração do psiquismo. Esta representação geral guia uma análise dos casos de quatro profissionais de saúde mental que colocaram suas profissões ao serviço de regimes autoritários latino-americanos para torturar presos políticos entre os anos 60 e 70 do século XX: o psiquiatra mexicano Salvador Roquet, o psicanalista brasileiro Amílcar Lobo Moreira, o psicólogo uruguaio Dolcey Brito e o psicólogo chileno Hernán Tuane.

Palavras-chave: Ditadura, Tortura, Psicologia, Psicanálise, Psiquiatria. 


\title{
Psychology and Destruction of the Psyche: the Professional use of Psychological Knowledge for Torture of Political Prisoners
}

\begin{abstract}
This article addresses the issue of the use of Psychology for the torture of political prisoners in the world and especially in Latin America. First, it examines the recent debate on psychologists involved in torture in the United States. Then it reminds the precedents of the use of Psychology to torture in Nazi Germany, Francoist Spain, French colonial repression in Algeria and US military strategy during the Cold War. The consideration of such precedents and the recent debate in the United States allows arriving at a general representation of the way in which Psychology operates in torture understood as a form of suppression and disintegration of the psyche. This general representation guides an analysis of the cases of four mental health professionals who put their professions at the service of Latin American authoritarian regimes to torture political prisoners between the 1960s and 1970s: the Mexican psychiatrist Salvador Roquet, the Brazilian psychoanalyst Amílcar Lobo Moreira, the Uruguayan psychologist Dolcey Brito and the Chilean psychologist Hernán Tuane.
\end{abstract}

Keywords: Dictatorship, Torture, Psychology, Psychoanalysis, Psychiatry.

\section{Introducción}

Al ocuparse de la relación entre la Psicología y la tortura, Suedfeld (1990) la concibió a través de tres posibles roles del psicólogo: el de torturado, el de torturador y el de agente externo que intenta comprender la tortura y tratar sus efectos en sus víctimas. Este último rol, el más natural según Suedfeld, es efectivamente el que suelen desempeñar los psicólogos en la variada literatura científica y académica sobre el tema. En sus reflexiones e investigaciones sobre la tortura, los profesionales de la Psicología no aparecen generalmente ni como víctimas ni como verdugos, sino como especialistas o como sanadores, como quienes comprenden o como quienes tratan.

En lo que se refiere a la comprensión psicológica de la tortura, se han considerado factores tan disímiles como el poder de la situación (Haney, Banks, \& Zimbardo, 1973), la obediencia a la autoridad por parte del torturador (Milgram, 1974), los mecanismos defensivos internos del propio torturador y el papel externo de la dominación y de las contradicciones sociales (Bendfeldt-Zachrisson, 1988), una hipotética destructividad humana extrema (Staub, 1990), diversos aspectos contextuales e ideológicos (Dobles, 1990), la influencia de los contextos sociales y de las presiones y prejuicios también sociales (Fiske, Harris, \& Cuddy, 2004), la falsa creencia popular en la eficacia de la tortura (Janoff-Bulman, 2007), la deshumanización de la víctima (Viki, Osgood, \& Phillips, 2013) y la personalidad autoritariay socialmente dominante (Lindén, Björklund, \& Bäckström, 2016). En lo concerniente al tratamiento psicológico, psicoterapéutico y psicosocial de los efectos de la tortura, tenemos valiosas propuestas generales (v.g. Gorman, 2001; Pope, \& Garcia-Peltoniemi, 1991; Wilson y Drozdek, 2004) y evaluaciones de las propuestas (v.g. Campbell, 2007; Patel, Willias, \& Kellezi, 2016), así como testimonios de intervenciones que se han realizado, por ejemplo, entre exiliados sudamericanos en Bélgica (González-Bermejo, 1979), entre víctimas de la represión política en Chile (Lira, \& Weinstein, 1984) y Guatemala (Hanscom, 2001), entre palestinos en Gaza (Qouta, \& El-Sarraj, 2002), entre los refugiados tibetanos en la India (Ketzer, \& Crescenzi, 2002) y entre víctimas de opositores maoístas y de fuerzas represivas gubernamentales en Nepal (Van Ommeren, Sharma, Prasain, \& Poudyal, 2002).

Además de hacer lo que suelen hacer, comprender y tratar los efectos de la tortura, puede ocurrir que los profesionales de la Psicología, siguiendo la distinción de Suedfeld, sean ellos mismos torturados o torturadores. El primer caso, ya estudiado en contextos como el brasileño (Carvalho, 2013) y el argentino (Carpintero, \& Vainer, 2005), pone a los psicólogos en la situación de cualquier víctima de tortura y quizás tan solo resulte significativo para la Psicología por lo que nos enseña sobre su historia, sobre su lugar en la 
sociedad y sobre los riesgos de algunas formas de ejercicio profesional. En cuanto al segundo caso, el de los psicólogos como quienes torturan o contribuyen de algún modo a torturar, tiene obviamente una gran significación para la Psicología, pero significativamente ha sido muy poco abordado en décadas anteriores, con la excepción de unos pocos estudios generales, como el clásico de Suedfeld (1990), y otros circunscritos a contextos particulares como el latinoamericano (v.g. Riquelme, 2004).

El desinterés por el tema es desconcertante cuando uno considera su gravedad y todo lo que puede revelarnos sobre la Psicología en sí misma, sobre la manera en que existe y opera, sobre su función en el sistema socioeconómico y su relación con el orden político establecido. Es precisamente por esto que aquí deseamos abordar el tema de los psicólogos torturadores. Y quizás haya sido por lo mismo, paradójicamente, que el tema fuera tan desatendido en el pasado, hasta que llegó el momento, hace poco, en que sencillamente ya no podía ignorarse más. Fue el momento en que estalló el escándalo por la participación de psicólogos estadounidenses en la tortura de sospechosos de terrorismo. Vimos entonces, de pronto, una avalancha de artículos acerca de algo muy viejo que salía súbitamente a la luz y parecía nuevo, inédito y sin precedentes (v.g. Arrigo, \& Long, 2008; Costanzo, Gerrity, \& Lykes, 2007; Lira, 2008; Saldarriaga, 2009; Soldz, 2008, 2011; Suedfeld, 2007; Welch, 2010).

Aunque tomando nuestras distancias con respecto al actual debate en torno a los psicólogos torturadores de los Estados Unidos, lo retomaremos aquí, de manera lateral, para problematizar algunos de sus términos y para cuestionar una posición en la que simultáneamente se ha disimulado y justificado el empleo de la Psicología para torturar. Luego, aportando una contextualización que suele faltar en el actual debate, recordaremos brevemente los precedentes de tal empleo en la Alemania nazi, la España franquista, la represión colonial francesa en Argelia y la estrategia militar estadounidense durante la Guerra Fría. La consideración de tales precedentes y del reciente escándalo en los Estados Unidos nos permitirá llegar a una representación general de la forma en que la Psicología opera en la tortura como forma de supresión y desintegración del psiquismo. Esta representación general guiará nuestro análisis de los casos de cuatro profesionales de la salud mental que pusieron sus profesiones al servicio de regímenes autorita- rios latinoamericanos para torturar a presos políticos entre los años 60 y 70 del siglo XX: primero el psiquiatra mexicano Salvador Roquet, al que le dedicaremos una sección completa por ser el primero en el tiempo y el menos conocido, y luego el psicoanalista brasileño Amílcar Lobo Moreira, el psicólogo uruguayo Dolcey Brito y el psicólogo chileno Hernán Tuane, de los que nos ocuparemos brevemente.

Los casos mencionados no podrán analizarse aquí de modo amplio y exhaustivo. Tendremos que ser muy selectivos y centrarnos tan solo en aquellos detalles con los que mejor pueda confirmarse, ilustrarse y problematizarse nuestra conjetura sobre la utilización de la Psicología para la destrucción de su propio objeto. Sin embargo, ante estos detalles, intentaremos dar voz a los involucrados, ofreciendo citas literales y favoreciendo las fuentes primarias. Nuestro análisis tendrá cierta densidad teórica y será de carácter crítico reflexivo y no solo histórico narrativo. En otras palabras, no solo buscará echar luz y atraer la atención sobre uno de los capítulos más sombríos de la historia de la Psicología en América Latina, sino que también intentará elucidar el encargo que la Psicología cumple al utilizarse para suprimir y desintegrar la esfera psíquica mediante la tortura. Esta elucidación, a su vez, debería servir para profundizar en todo lo que está en juego en el actual debate sobre los psicólogos torturadores en los Estados Unidos.

\section{Mitchell, Jessen and Associates: una compañía de Psicología especializada en la tortura}

En diciembre de 2014, en el Senado de los Estados Unidos, el Comité Selecto sobre Inteligencia hizo público un informe sobre la tortura de sospechosos de terrorismo por parte de la Agencia Central de Inteligencia (CIA) durante la presidencia de George W. Bush (Senate Select Committee on Intelligence, 2014). El informe divulgaba la participación de psicólogos en el diseño y la implementación de "técnicas de interrogación mejorada" que se utilizaban en centros clandestinos de detención de la CIA alrededor del mundo, entre ellos Bagram, Guantánamo y Abu Ghraib, y que incluían diversas formas de tortura, entre ellas las posiciones corporales incómodas prolongadas, la exposición a un frío intenso o a ruidos ensordecedores, el ahogamiento simulado, la privación sensorial, la privación de sueño hasta el punto de provocar alucinaciones, la privación de alimentos 
y bebidas, la rehidratación anal y el confinamiento en estrechas cajas similares a ataúdes. El parágrafo 13 del mismo informe detallaba cómo la CIA contrató especialmente a dos psicólogos, fundadores de una compañía privada especializada en "interrogaciones mejoradas", a quienes pagó 80 millones de dólares para "desarrollar, operar y evaluar las operaciones de interrogación", lo que habían hecho "basándose en la indefensión aprendida" (Senate Select Committee on Intelligence, 2014, p. 11).

Al mencionar la "indefensión aprendida" (learned helplessness), el informe del Comité Secreto del Senado se estaba refiriendo a un concepto que le aseguró la celebridad al psicólogo estadounidense Martin Seligman, promotor de la Psicología positiva y antiguo presidente de la Asociación Americana de Psicología (APA). El concepto de Seligman (1975) describe la condición de quien aprende a sentirse impotente y a comportarse pasivamente por causa de circunstancias como los castigos continuos. Las torturas, operando como castigos continuos, harían "aprender la indefensión" a los sospechosos de terrorismo, los cuales, convertidos en seres indefensos, impotentes y pasivos, habrían de mostrarse lógicamente más dóciles y sumisos en el curso de sus interrogatorios. Al menos esto era lo que se esperaba, pero no fue lo que ocurrió, como lo muestra el informe del Comité Secreto del Senado, en el que se denuncia la ineficacia de la estrategia dirigida por los dos psicólogos seguidores de Seligman.

El Comité Secreto había cubierto bajo el anonimato a los dos psicólogos que dirigían las torturas de la CIA, pero los medios no tardaron en identificarlos y en difundir sus nombres: James Elmer Mitchell y John Bruce Jessen (Windrem, 2014). La identificación, por cierto, no podía ser más fácil. Ambos psicólogos ya habían sido públicamente denunciados mucho tiempo antes. Eran tan conocidos entre los profesionales de la Psicología, que "la sola reacción ante sus nombres se había convertido en la prueba de fuego de la actitud ante la coerción y los derechos humanos" (Eban, 2007, párr. 18).

Mitchell y Jessen estaban en el centro de una enardecida controversia que se había desatado por una resolución de la APA, en julio 2005, en la que se autorizaba que los psicólogos rindieran sus servicios en los interrogatorios militares. En el contexto de esta polémica, el informe del Comité Secreto de 2014 vino a justificar, validar y reforzar la posición de quienes, como Costanzo et al. (2007), habían exigido a la APA una "condena clara", una "investigación independiente" y una "prohibición expresa" de la participación de psicólogos en "torturas o en otras formas de tratamiento cruel, inhumano o degradante como técnica de interrogación" (p. 10). El mismo informe sirvió para que aprendiéramos a desconfiar de quienes, como Suedfeld (2007), ponían en duda que los profesionales de la Psicología estuvieran verdaderamente implicados en "inventar o aplicar técnicas de tortura" (p. 59), pero al mismo tiempo recomendaban a la APA, con una buena dosis de cinismo, que alentase a los psicólogos a que ayudaran a "autoridades legítimas a evitar falsas confesiones y a obtener información veraz con el nivel mínimo posible de incomodidad, privación o dolor (mental o físico) de las personas interrogadas" (p. 61).

Lo que Suedfeld plantea es bastante claro: aun cuando las policías de los gobiernos instituidos emplearan el dolor para extraer información, aun cuando recurrieran a la tortura en sus interrogatorios, deberían ser apoyadas por los profesionales de la Psicología. O peor aún: había que estimular a los psicólogos a poner su profesión al servicio de los torturadores en los interrogatorios, pero siempre y cuando la tortura fuera lo menos dolorosa posible. Sobra decir que "lo menos dolorosa posible" puede ser sinónimo de "inmensamente dolorosa" cuando se trata de personas particularmente refractarias al interrogatorio. ¿Pero acaso estas personas no son precisamente aquellas que necesitan ser torturadas? Pedir que "se les torture lo menos posible para conseguir lo que se necesita" es igual a pedir que "se les torture solamente lo que se necesita". El razonamiento de Suedfeld es el de cualquier torturador simplón y suficientemente sensato: si ya hemos obtenido todas las informaciones que necesitábamos, ¿para qué molestar al torturado y para qué molestarse uno mismo al intensificar la tortura?

Como hemos visto, Suedfeld se empeña en disimular y justificar la tortura con los argumentos del más vulgar de los golpeadores. Esto es todo lo que hace con lo que él mismo describe, con un orgullo enternecedor, como su "pensamiento complejo" (Suedfeld, 2007, pp. 55-56). Tal pensamiento es el mismo con el cual, 17 años antes, abordó la relación de la Psicología con la tortura, llegando al reconfortante descubrimiento de que tan solo existía un caso demostrado en el que un psicólogo profesional hubiera practicado la tortura (Suedfeld, 1990). Podía entonces concluirse alegremente que la Psicología no 
es de ningún modo, por así decir, una "profesión útil para torturar". Sin embargo, para demostrar lo contrario, ahí están los psicólogos James Elmer Mitchell y John Bruce Jessen con sus colaboradores, entre ellos el mismísimo expresidente de la APA Joseph Matarazzo, y con su firma Mitchell, Jessen and Associates: no solo una macabra "empresa de tortura", sino una "compañía especializada en la Psicología para la tortura". De modo que ya no es verdad que haya solo un caso de psicólogo torturador en el mundo. Pero de cualquier manera, como lo veremos en las siguientes páginas, tampoco era verdad en 1990, cuando Suedfeld lo afirmaba. Y, para empezar, ipor qué reducir la cuestión del uso profesional de la Psicología para la tortura a la simple constatación de casos plenamente demostrados en los que haya un profesionista psicólogo torturador? Tal reducción resulta inadmisible por muchas razones, entre ellas tres bastante obvias: 1) la tortura, especialmente la psicológica, no suele ser algo plenamente demostrable; 2) los psicólogos pueden contribuir a la tortura de modo indirecto, inspirándola o concibiéndola, sin participar directamente en ella; y 3) no son únicamente los psicólogos, sino también los psiquiatras, los psicoanalistas y otros profesionales de la salud mental, quienes están en condiciones de hacer un uso profesional, relativamente riguroso y metódico, de la Psicología como ciencia para torturar.

Lo cierto es que hay varias situaciones concretas conocidas, algunas de ellas bien evidenciadas y otras bastante verosímiles, en las que el conocimiento científico psicológico ha sido utilizado profesionalmente por psicólogos y otros especialistas de la salud mental para inspirar, asesorar, concebir, diseñar, perfeccionar o aplicar técnicas de tortura. Semejante utilización de la Psicología será ejemplificada y examinada más adelante a través de los ya mencionados cuatro casos latinoamericanos de la segunda mitad del siglo XX. Sin embargo, antes de pasar al examen puntual de estos casos, conviene que nos detengamos un momento en los precedentes del empleo de la Psicología para la tortura y en algunas consideraciones generales en torno a tal empleo.

\section{Breve revisión histórica de la Psicología en la tortura: nazismo alemán, franquismo español, colonialismo francés e imperialismo estadounidense}

A finales de los treinta y principios de los 1940, entre los franquistas españoles y los nazis alemanes, encontramos ya claros indicios de vinculación entre la tortura y la Psicología. Sabemos que la Gestapo recurrió a diversos métodos rigurosos de "interrogatorio intensificado" (verschärfte Vernehmung) que eran idénticos o muy similares a las "técnicas de interrogación mejorada" propuestas por Mitchell y Jessen. La exposición al frío y la privación sensorial, de sueño, de alimento y bebida, formaban parte del arsenal de estrategias empleadas por los nazis en los interrogatorios durante la Segunda Guerra Mundial. Un caso bien documentado es el de Richard Wilhelm Hermann Bruns, Rudolf Theodor Adolf Schubert y Emil Clemens, quienes fueron juzgados en Noruega por haber efectuado "interrogatorios intensificados" entre 1942 y 1945, durante la ocupación alemana de aquel país (Sullivan, 2009; Suprema Corte de Noruega, 1946).

Mientras la Gestapo se valía de un método psicológico de tortura para interrogar a posibles opositores al nazismo, centenares de niños, adolescentes $\mathrm{y}$ algunos adultos eran torturados por profesionales de la salud mental en diversas clínicas psiquiátricas y "guarderías para niños especiales" en Alemania y en las zonas ocupadas por los nazis. Es bien conocido el caso del hospital Am Steinhof y específicamente de la clínica Am Spiegelgrund de Viena, en donde el psiquiatra Heinrich Gross no solo decidió la muerte de muchos internos, sino que también los hizo pasar por los más dolorosos tormentos con fines de reeducación y experimentación (Czech, 2014; Jahn, 2012; Martens, 2004). Poco tiempo antes, Robert Ritter, doctor en Psicología educativa, se había dedicado a realizar extensas investigaciones para demostrar, según él, que los gitanos eran congénitamente "criminales y asociales", con lo que proporcionó la justificación psicológica perfecta que se necesitaba para internarlos en campos de concentración y ahí someterlos a un trato inhumano que hoy correspondería exactamente a lo que entendemos por tortura (Friedlander, 1995, p. 252).

El trato recibido por los gitanos en la Alemania nazi es comparable al que recibieron por la misma época muchos presos políticos en la España franquista. Aquí también se contó con una justificación psicológica perfecta, la ofrecida por el psiquiatra Antonio Vallejo Nájera, jefe de un Gabinete de Investigaciones Psicológicas dedicado a investigar las raíces psíquicas del marxismo. Vallejo Nájera pretendió probar científicamente que los marxistas eran "psicópatas antisociales" que debían segregarse para "liberar a la sociedad de plaga tan temible” (Vallejo Nájera, 1939, p. 52). Esta forma de "terapia segregacionista" buscaba una 
"liquidación moral" de los enemigos comunistas, los cuales, en los propios términos de Vallejo Nájera, "perderán la libertad, gemirán durante años en prisiones, purgando sus delitos" (Vinyes Ribas, 2001, pp. 237239). El psiquiatra franquista parece confesar aquí, de manera un tanto velada, un objetivo de infligir dolor que es propio de la tortura y que puede servir por sí mismo para justificar su necesidad como una forma de expiación, incluso en ausencia de cualquier otro propósito. De cualquier modo, aun cuando Vallejo Nájera no hubiera justificado abiertamente la tortura de los comunistas como algo necesario, sus conclusiones sí podían servir y seguramente sirvieron para excusarla como algo moralmente aceptable, ya que presentaban a la víctima como un "infra-hombre malvado sobre el cual todo era lícito” (p. 240).

Después de haber sido una tarea obsesiva de nazis y franquistas como Vallejo Nájera, la guerra contra los comunistas pasó a ser una de las ocupaciones principales del gobierno de los Estados Unidos. Fue muy pronto, ya en los años 50 del siglo XX, poco después del fin de la Segunda Guerra Mundial, cuando la CIA empezó a servirse de psicólogos y psiquiatras en su desarrollo de técnicas de tortura dirigidas especialmente contra sospechosos de comunismo o de espionaje de los países comunistas. Hoy en día, gracias a las investigaciones exhaustivas de McCoy (2006), sabemos que la agencia de inteligencia estadounidense, a través de su programa de interrogatorios alternativos denominado "MKUltra", utilizó intensivamente, a menudo financió y a veces dirigió el trabajo de algunas de las más importantes figuras de la Psicología y la psiquiatría entre los años 50 y 60 del siglo XX.

Las investigaciones experimentales del psicólogo Donald O. Hebb (1904-1985) sobre la "privación sensorial" en la Universidad de McGill, financiadas por la CIA y efectuadas a partir de 1951 con animales y con humanos voluntarios a los que se remuneraba, demostraron que la eliminación de estímulos visuales, auditivos y táctiles podía posibilitar el "lavado de cerebro" y el "control de la mente" al provocar una "ruptura de la actividad organizada en los procesos centrales complejos" del cerebro (McCoy, 2006, pp. 41-42). En la misma Universidad de McGill, entre 1957 y 1963, los experimentos particularmente violentos del psiquiatra Donald Ewen Cameron, dirigidos y no solo financiados por la CIA, ya no se limitaban a las condiciones de posibilidad del lavado de cerebro y el control de la mente, sino que realizaban en acto y de manera forzada este lavado y este control a través de lo que el propio Cameron denominaba "conducción psíquica” (psychic driving): una estrategia que se valía de electrochoques, drogas y "repetición de señales verbales" para desintegrar y reprogramar el psiquismo de pacientes en un hospital psiquiátrico (pp. 43-44). Desde el año de 1956, los psiquiatras y neurólogos Lawrence Hinkle y Harold Wolff, del Centro Médico de Cornell y también con financiamiento y dirección de la CIA, cuestionaron el uso de técnicas de lavado de cerebro y de control de la mente en los interrogatorios, y pusieron en evidencia la mayor eficacia de "prácticas policiales" tradicionales como el "aislamiento", la "incomodidad" y otros motivos de "dolor auto-infligido" en los que el malestar procedería del mismo sujeto, de su cuerpo y de su mente, de su posición incómoda y de sus ideas, en lugar de provenir de un agente externo, haciendo que el torturado se percibiera como responsable de la tortura, escindiéndolo de sí mismo y evitándose así que se acentuara su resistencia contra los torturadores (pp. 45-46). Por último, en 1961, en la Universidad de Yale y con posible financiamiento de la CIA, los experimentos de Stanley Milgram, demostrando que la gente común era capaz de torturar y hasta matar por obedecer a la autoridad, sirvieron para convencer a los servicios de inteligencia estadounidenses que podían valerse de cualquier "soldado o policía ordinario" para ser obedecidos y llevar a cabo las torturas diseñadas gracias a las investigaciones de Hebb, Cameron, Hinkle y Wolff (pp. 47-49).

En los años 1950, mientras los recién mencionados investigadores trabajaban de manera voluntaria o involuntaria para la CIA en clínicas y laboratorios de los Estados Unidos, algunos psiquiatras franceses administraban sustancias psicoactivas para obtener informaciones de los militantes políticos torturados en el contexto de la Guerra de Argelia. Fanon (1965) relata cómo estos "sueros de la verdad", que provocaban "pérdida de control" y "embotamiento de la conciencia", dejaban graves secuelas como la "incapacidad para distinguir lo verdadero de lo falso" y un "temor casi obsesivo a decir lo que debe mantenerse oculto” (pp. 137-138). Aún peores eran las secuelas dejadas por los electrochoques, también usados por los psiquiatras que trabajaban para los centros franceses de tortura en Argelia, como lo sabemos por el mismo Fanon. Al mismo tiempo que Donald Ewen Cameron buscaba desintegrar el psiquismo con descargas eléctricas, los psiquiatras franceses usaban la misma técnica en los interrogatorios para causar la “confusión, la relajación de la resistencia y la desa- 
parición de las defensas" del torturado, haciendo que al final solo quedara una "personalidad en pedazos" cuya recomposición y rehabilitación era "extremadamente difícil" (p. 138).

De los expertos franceses en tortura durante la Guerra de Argelia, el más polémico fue Paul Aussaresses, quien enseñó técnicas de contrainsurgencia en Fort Bragg y Fort Benning, en los Estados Unidos, entre 1961 y 1962 (Robin, 2003). Posteriormente, en los años 1970, mientras asesoraba la dictadura brasileña, Aussaresses ofreció el mismo entrenamiento a oficiales brasileños, chilenos, argentinos y venezolanos en el Centro de Instrucción de Guerra en la Selva (Centro de Instrução de Guerra na Selva). Mientras que Aussaresses entrenaba directamente a futuros torturadores, otros dos expertos franceses, David Galula y Roger Trinquier, escribían textos que se convertirían en manuales obligados en los centros de formación en contrainsurgencia en los Estados Unidos y en América Latina. Tenemos aquí algunos principios generales para encuadrar la tortura: si Galula considera que sería "peligroso y contraproducente dejar los interrogatorios a aficionados", Trinquier piensa que "los especialistas deberán, por todos los medios, arrancar los secretos" del torturado, quien "deberá entonces, como el soldado, enfrentar el sufrimiento y quizás la muerte" (Le Cudennec, 2009, párr. 3). El trabajo más influyente de Trinquier, "La guerra moderna", obtenido por la CIA a través de la mediación del propio Aussaresses, terminó convirtiéndose en una referencia obligada para la inteligencia estadounidense y orientó la estrategia en una Guerra de Vietnam en donde los comandos terminaron "actuando exactamente como los escuadrones de la muerte de Paul Aussaresses" en Argelia (Robin, 2003, p. 254).

Tras diez años de observaciones o reflexiones de expertos franceses y de experimentos de investigadores estadounidenses, la CIA pudo elaborar por fin, en 1963, el famoso "Manual de Interrogación de Contrainteligencia KUBARK", en el que la agencia estadounidense de inteligencia ofrecía un amplio abanico de avanzadas técnicas de tortura, no sin antes dar crédito a la "importancia y relevancia" del trabajo de los "psicólogos americanos" cuyas "investigaciones psicológicas" y "hallazgos psicológicos" habían generado el "conocimiento pertinente, moderno", en el que se basaban las técnicas propuestas (CIA, 1963, p. 2). La insistencia en la Psicología es aquí notable, así como también lo es la centralidad de la privación sensorial de Hebb, la opción por el dolor auto-infligido de Hinkle y Wolff, y la forma en que las diversas "técnicas de coerción" están invariablemente "diseñadas para inducir una regresión" en la que el torturado, perdiendo la estructura psíquica y las "defensas del hombre civilizado", termina viendo al interrogador como una "figura paterna" a la que debe someterse (pp. 83, 90).

El manual KUBARK, destilado sintético del paradigma psicológico de tortura de la CIA, fue utilizado entre los años 1970 y 1980 en los diferentes campos de batalla de la Guerra Fría, entre ellos el de Vietnam, en el que los métodos para torturar en los interrogatorios, como nos lo recuerda McCoy (2009), terminaron traduciéndose en "la cruda brutalidad física del programa Phoenix que produjo 46.000 ejecuciones extrajudiciales y poca inteligencia accionable" (párr, 16). En 1983, exactamente 20 años después del lanzamiento del KUBARK y sobre la base del mismo conocimiento psicológico, empezó a circular un segundo manual de tortura e interrogatorio, el Human Resource Exploitation Training Manual, que estaba especialmente diseñado para el contexto latinoamericano y que se utilizó en campos estadounidenses de entrenamiento en Honduras. El nuevo manual exhortaba a "manipular el ambiente del sujeto para crear situaciones desagradables o intolerables" para él, así como hacerle creer que sus familiares estarían "sufriendo o en peligro" (Cohn, Thompson, \& Matthews, 1997, párr. 11, 15). Al igual que en el KUBARK, se buscaba desencadenar procesos regresivos, recomendando "tener a un psicólogo disponible cuando se induce la regresión” (párr. 37).

Tras el final de la Guerra Fría, en 1994, los Estados Unidos ratificaron la Convención contra la Tortura de la ONU. Sin embargo, al definirse las formas de tortura en el documento oficial de ratificación enviado por el presidente Bill Clinton al Congreso, no se consideraba ni la privación sensorial de Hebb ni el dolor auto-infligido de Hinkle y Wolff, es decir, el eje rector de la tortura psicológica, psicológicamente fundamentada e implementada, que la CIA desarrolló en los años 1950 (McCoy, 2009). Es así como se ha mantenido un vacío legal por el que se ha posibilitado que esta forma de empleo de la Psicología para la tortura se perpetúe desde los años 70 del siglo XX hasta el escándalo de Mitchell y Jessen entre 2007 y 2014.

\section{La Psicología como dispositivo destructivo}

La revisión histórica recién ofrecida nos ha permitido apreciar dos grandes usos de la Psicología para los torturadores: la justificación de la tortura, para Vallejo 
Nájera, Ritter y quizás Gross, y la concepción y realización de la tortura, para todos los demás. El segundo uso presupone de algún modo el primero, pues la tortura no se realiza y se concibe psicológicamente sino con un objetivo también psicológico por el que se justifica su eficacia en la misma esfera de la Psicología. Es verdad que el objetivo último no es exactamente psicológico, pues consiste en la obtención de la verdad en los interrogatorios, pero este objetivo mediato se alcanza invariablemente a través de un propósito psicológico inmediato: la coerción y la resultante regresión del torturado, su anulación o sometimiento, la inhabilitación de su voluntad, la supresión de su personalidad, la neutralización de sus resistencias o de sus defensas, la desintegración o desorganización y la sucesiva reorganización o reprogramación de su psiquismo. Todos estos fines psicológicos justifican psicológicamente la tortura como un medio eficaz para alcanzarlos y también para alcanzar, a través de ellos, la obtención de la verdad en el interrogatorio. A veces incluso la obtención de la verdad termina perdiéndose de vista, como en el caso de Cameron, para quien la reprogramación del psiquismo aparece como un fin en sí mismo.

Ya sea que el fin último sea reorganizar el psiquismo u obtener la verdad o incluso castigar o reeducar al sujeto, el objetivo inmediato de la tortura será generalmente negativo y consistirá las más de las veces en desorganizar, desintegrar, neutralizar, anular, someter, inhabilitar, suprimir, destruir. Este objetivo destructivo podrá estar subordinado a uno reconstructivo, desde luego, pero la reconstrucción requiere de otros medios que la tortura, la cual, por sí misma, tal como se concibe y se realiza en la Psicología, suele tener un objetivo inmediato eminentemente destructivo. La destrucción habrá de revestir las más diversas formas: la ruptura de la actividad cerebral organizada a través de la privación sensorial de Hebb, la desintegración del psiquismo en la conducción psíquica de Cameron, la escisión del sujeto en el dolor auto-infligido de Hinkle y Wolff, la pérdida del control y el embotamiento de la conciencia por los sueros de la verdad de los psiquiatras franceses en Argelia, el despedazamiento de la personalidad por los electrochoques administrados por los mismos psiquiatras franceses, la regresión y la desaparición de las defensas del hombre civilizado en el manual KUBARK.

En los casos recién mencionados, la tortura opera como una estrategia psicológica, psicológicamente concebida y realizada, para conseguir la destrucción psíquica, personal y subjetiva, de quien es torturado. Vislumbramos aquí un propósito destructivo de la Psicología que debe sumarse a sus otros fines positivos mejor conocidos, particularmente el ideológico, enfatizado por los marxistas (v.g. Braunstein, Pasternac, Benedito, \& Saal, 2006; Parker, 2010), y el disciplinario, en el que insisten los foucaultianos (v.g. Rose, 1989, 1998). De pronto nos percatamos de que la Psicología no debe dedicarse únicamente a ideologizar y disciplinar, a reproducir de este modo su propio objeto y el sistema en el que se inserta, es decir, a formar y conformar una subjetividad recluida en su interioridad individual y relacionada exteriormente con los otros de cierto modo y en cierta estructura transindividual. Además de sus funciones reproductivas, la Psicología nos muestra de pronto una función destructiva, en la cual, paradójicamente, la ciencia del psiquismo destruye su propio objeto psíquico. Esta destrucción parece derivar directamente, ya no de los aparatos ideológicos y disciplinarios del Estado, sino de sus aparatos represivos: de la violencia directa y no de la indirecta, de la dominación y no de la persuasión, de la tiranía y no de la hegemonía.

\section{Salvador Roquet: la tortura psicosintética en la dictadura perfecta mexicana}

El siglo XX convirtió a México en un lugar de acogida para perseguidos políticos: en los años 1930, españoles que huían del franquismo; en los 1970, argentinos, chilenos y otros que escapaban de las dictaduras del cono sur; en los 1980, guatemaltecos y salvadoreños que habían sufrido la violencia de las tiranías sostenidas por el imperialismo estadounidense. Para estos exiliados y para muchos otros, México representaba un espacio de tolerancia y libertad. Era un refugio para los demócratas del mundo entero, así como para los ideales democráticos. El gobierno mexicano llegó incluso a ser percibido como la democracia perfecta de América Latina. Sin embargo, especialmente en la segunda mitad del siglo XX, la realidad era otra, completamente diferente.

En 1990, durante un encuentro de intelectuales transmitido por la televisión mexicana, el escritor peruano Vargas Llosa (1990, 1 de septiembre) no dudó en afirmar que México, lejos de ser la democracia perfecta, era la "dictadura perfecta", una "dictadura sui generis que muchos en América Latina habían tratado de emular", una "dictadura camuflada" que 
tendría "todas las características de una dictadura", entre ellas "la permanencia, no de un hombre, pero sí de un partido inamovible” (párr. 3-5). Además de la permanencia del Partido Revolucionario Institucional (PRI) que gobernó ininterrumpidamente México desde 1928 hasta el año 2000, una característica típicamente dictatorial por la que se distinguió el régimen gubernamental mexicano fue la represión brutal, sistemática y permanente contra sus opositores. Esta estrategia represiva incluyó el encarcelamiento, la tortura, la desaparición y el asesinato de miles de personas, así como importantes matanzas colectivas, en grupo y hasta en masa, como la de henriquistas en La Alameda en 1952 (unos 200 muertos), las de la Asociación Cívica Guerrerense en Chilpancingo en 1960 y en Iguala en 1962 (más de 50 víctimas en total), las de copreros en Acapulco (entre 30 y 80 muertos y desaparecidos) y maestros y padres de familia en Atoyac en 1967 (5 muertos), Tlatelolco en 1968 (entre 100 y 300 muertos), el Halconazo en 1971 (entre 30 y 50 muertos), La Trinidad en 1982 (9 muertos), Aguas Blancas en 1995 (17 muertos), Acteal en 1997 (45 muertos) y El Charco en 1998 (11 muertos), por mencionar únicamente las más conocidas.

Entre finales de los sesenta y principios de los 1970, en uno de los períodos más sangrientos de la dictadura del PRI en México, la estrategia represiva gubernamental se convirtió en una guerra de exterminio en la que destacó la figura siniestra de Miguel Nazar Haro (1924-2012), máxima autoridad en la Dirección Federal de Seguridad (DFS) y feroz anticomunista formado por militares estadounidenses en la Escuela de las Américas de Panamá (López de la Torre, 2013; Rodríguez Castañeda, 2013; Torres, 2008). El equipo de Nazar Haro estaba formado por decenas de esbirros, matones y torturadores sin formación alguna, pero también por algunos profesionales acreditados, como el psiquiatra Salvador Roquet Pérez (1920-1995). Este controvertido psiquiatra, de hecho, se había ganado cierta celebridad en México y en el mundo, hasta el punto de ser comparado con Freud por algunos de sus admiradores, gracias a su invención y desarrollo de la "psicosíntesis": un tratamiento psicoterapéutico apoyado en alucinógenos como peyote, hongos, datura y LSD (Ramírez, 1985) Fue precisamente una variante del método psicosintético lo que Roquet parece haber transformado, a finales de los sesenta, en la elaborada técnica de tortura psicológica implementada en militantes políticos deteni- dos por la DFS, entre ellos un dirigente estudiantil del movimiento de 1968, el maoísta Federico Emery Ulloa (Marín, 1985, 28 de marzo).

Es por Emery, por su testimonio detallado vertido a través de varias entrevistas y de una denuncia penal, por quien ahora podemos reconstruir con cierta exactitud la forma $\mathrm{o}$ al menos una de las formas en que Roquet operaba. Su intervención no se realizaba en la famosa prisión de Lecumberri en la que se concentraban los presos políticos, sino en una casa de seguridad a la que se desplazaba únicamente a quien pasaría por la tortura psicosintética. El preso, forzado a ingerir diversos alucinógenos químicos y naturales, se encontraba encerrado en un cuarto amplio, de aproximadamente seis por ocho metros, en el que había dos sillas y una mesa con una grabadora y con dos proyectores, uno de cine de 16 milímetros y otro de diapositivas, frente a dos pantallas.

Durante el proceso, que se prolongada por $10 \mathrm{a}$ 20 horas, el preso debía escuchar "música de Wagner a todo volumen", hasta el punto de "lastimar los oídos", mientras veía "películas pornográficas" de "lesbianas y orgías" que se entremezclaban con diapositivas de "pinturas realizadas por locos", por "locos de manicomio", según se lo explicó el propio Roquet a Emery (Marín, 1985, 29 de marzo, párr. 5; Monge, 2002, párr. 16). Las drogas consumidas por el sujeto lo hacían "pasar de la histeria al terror" (Sánchez, 2003, párr. 1), u oscilar entre "el miedo, el miedo, el miedo", y "el coraje hasta tratar de destruir", y "luego la euforia, la alegría hasta la risa y la carcajada" (Marín, 1985, 29 de marzo, párr. 4). Finalmente, el sujeto "se hundía" y sentía un "temor pavoroso" que lo hacía correr hasta un rincón y tirarse en el suelo, y es entonces cuando Roquet, mostrándose amenazante y diciéndole "tú eres ratón, yo soy gato", podía empezar un largo interrogatorio de unas diez horas con centenares de preguntas centradas en las actividades políticas del torturado (Monge, 2002, párr. 17).

Emery no solo debió sufrir la psicosíntesis recién relatada, sino también torturas psicológicas más convencionales, entre ellas tres simulacros de ejecución en los que era conducido a lugares apartados, le ponían el cañón de un arma en la cabeza y jalaban el gatillo sin disparar. Las torturas psicológicas se complementaban, además, en este caso como en otros, con las más diversas torturas físicas, sin contar el aislamiento, las pésimas condiciones de encarcelamiento y el temor por la propia vida y por los seres queridos. 
Interpretando retrospectivamente lo ocurrido, Emery concluye que Nazar Haro, el director de la DFS, "quería llevarlo al borde de la locura, quería desacomodar sus sensaciones, sus percepciones, para acomodarlas a su modo" (Monge, 2002, párr. 27). No hay manera de saber cómo es que Emery llegó a esta conclusión, pero su interpretación resulta interesante por la manera en que relaciona la desorganización y la reorganización de los elementos psíquicos, es decir, en definitiva, las funciones destructiva y reconstructiva del psiquismo que distinguimos anteriormente.

La experiencia de Emery, tal como él mismola percibe, corresponde exactamente al proceso de reprogramación del psiquismo desarrollado por Cameron en el marco del ya mencionado programa MKUltra de la CIA. ¿Cómo no suponer que hay un vínculo entre este programa, en el que también se emplearon alucinógenos con el mismo propósito de obtener información, y la técnica de tortura psicosintética implementada por Roquet en el tiempo en el que trabajó para Nazar Haro? Tal suposición bien puede convertirse en convicción, especialmente cuando consideramos, por un lado, que el trabajo mexicano, realizado a finales de los sesenta, coincide temporalmente con el tiempo de operación de MKUltra entre 1953 y 1973, y, por otro lado, que Nazar Haro era un agente activo de la CIA al que la propia agencia protegió legalmente en los Estados Unidos (Torres, 2008), liberándolo de prisión, dándole inmunidad y presentándolo como un "contacto esencial de la CIA en México” (Scott, 2014, p. 46).

En lo que se refiere a Roquet, Emery terminó convenciéndose de que trabajaba para la CIA después de que fuera encarcelado, según el informe oficial, por su utilización psicoterapéutica de alucinógenos, pero justo en el momento en que el gobierno mexicano investigaba la presencia de la CIA en México (Marín, 1985, 29 de marzo). Esta versión es exactamente la inversa de otra, quizás menos verosímil, según la cual Roquet habría sido encarcelado precisamente por negarse a trabajar con la CIA, que le proponía instalarse en los Estados Unidos y "tratar" a "pacientes especiales" para el gobierno estadounidense (Wolfson, 2014, p. 171). En cualquier caso, independientemente de sus posibles relaciones con la CIA y de su indiscutible trabajo de torturador en la DFS, Roquet parece haber sido alguien bastante conservador que mostraba incomprensión y quizás incluso aversión hacia los jóvenes militantes de izquierda, psicopatologizándolos al atribuirles un "comportamiento pato- lógico autodestructivo" e incluyéndolos en la misma categoría que los hippies drogodependientes con sus "neurosis subyacentes" (Dawson, 2015, p. 126). Esta psicopatologización de la militancia revolucionaria de izquierda - que nos recuerda evidentemente a Vallejo Nájera -, junto con la misión curativa que el propio Roquet parece haberse fijado, han hecho que se llegue a considerar que él mismo no entendía su trabajo en la DFS como una simple "tortura" de perseguidos políticos, sino como un "tratamiento" de enfermos autodestructivos (p. 127).

El propósito de Roquet habría sido clínico, psicoterapéutico y no solo político. Se trataba de curar a los militantes y no solo de violentarlos, atormentarlos, vulnerabilizarlos y extraer información. Tal vez podamos conjeturar que el propósito final de la tortura, tal como Roquet la concebía, no era destruir al sujeto y así descubrir los secretos de su militancia revolucionaria, sino reconstruirlo y así curarlo de su enfermedad militante. Aunque esto quizás contradiga tanto la falta de cualquier indicio de reconstrucción como el peso del interrogatorio en la sesión de tortura psicosintética relatada por Emery, al mismo tiempo coincide con la interpretación retrospectiva del propio Emery, quien veía en su experiencia, como recordaremos, una desorganización para la reorganización del psiquismo. La reconstrucción y la curación habrían sido, pues, desde este punto de vista, el sentido último de la destrucción. En los términos mismos de Roquet (1971), el objetivo de la "desintegración" habría sido la "psicosíntesis", la “síntesis", la "reestructuración”, la “integración con los elementos esenciales” (párr. 11-14). Volvemos así al método propuesto por Cameron.

\section{Amílcar Lobo, Dolcey Brito y Hernán Tuane}

De los dos momentos sucesivos de la conducción psíquica de Cameron, el más característico es el segundo, el reconstructivo, pues ya hemos visto que el primero, el destructivo, suele aparecer como propósito inmediato de casi cualquier estrategia psicológica de tortura. Este propósito destructivo será el más visible en las estrategias de los tres profesionales torturadores de los que ahora nos ocuparemos: el médico y psicoanalista brasileño Amílcar Lobo, el psicólogo uruguayo Dolcey Brito y el psicólogo chileno Hernán Tuane Escaff, quienes aportaron sus conocimientos psicológicos para torturar a presos políticos en dictaduras del Cono Sur. Los tres empezaron a operar como torturadores justo después de Roquet, en los años 70 del siglo XX: Lobo en 1970, Dolcey en 1972 y 
Tuane en 1974. De los tres casos, quizás el más original y también el peor documentado sea el de Tuane, quien urdió una guerra psicológica en la que detectamos elementos propios de la intervención de la Psicología en la tortura, entre ellos el más fundamental, el destructivo, y quizás también el reconstructivo que ya hemos visto operar en Cameron y en Roquet.

El caso mejor conocido es el de Amílcar Lobo Moreira (1939-1997), el cual, entre 1970 y 1974, siendo médico, psicoanalista y miembro en formación de la Sociedad de Psicoanálisis de Río de Janeiro, participó activamente en sesiones de tortura en las que debía supervisar el estado físico de cada torturado (Lobo 1998). Su función precisa era informar si la víctima podía seguir siendo torturada, si "estaba fingiendo" y si "aún aguantaba”, no solo en sesiones ordinarias de tortura con fines de interrogatorio, sino también en "clases de tortura" con fines didácticos (CNV, 2014, pp. $351,355)$. Sin embargo, además de cumplir esta función, Lobo fue denunciado por intervenir en las torturas, por administrar sustancias psicoactivas y quizás también electrochoques. Una prisionera lo acusó de "aplicar algunas inyecciones" del más clásico suero de la verdad, el pentotal, para uno de los interrogatorios (Romeu, 2011, párr. 38). Otro torturado también cuenta cómo Lobo "aplicó pentotal muy lentamente" para sumirlo en "turbación mental y somnolencia" en los interrogatorios (Brasil, 2014, p. 370). En el mismo sentido, pero en una acusación más grave, el testimonio de una exguerrillera relata cómo Lobo habría sido "el jefe de su tortura", cómo la "llenaba de remedios psiquiátricos" que le hacían perder "la noción de tiempo, de calor, de frío", además de que se le hacía pasar por "simulaciones de ejecución" y por "descargas eléctricas" que "contraían la musculatura", todo en una estrategia "muy bien articulada para enloquecer" (Magalhães, 2009, párr. 215).

El objetivo de enloquecer a los torturados fue también denunciado al describir las estrategias de tortura diseñadas e implementadas en un centro de reclusión de Uruguay, a partir de 1972, por Dolcey Marcelino Brito Puig (1930-2016). Este psicólogo con "mediocre formación académica", graduado en una institución privada de "bien pobre reputación" (González Bermejo, 1985, pp. 108-109), habría convertido el Penal de Libertad en un "gran centro de enloquecimiento de presos políticos" (Uruguai, 1989, p. 221). Todo en el Penal parecía estar destinado a la "destrucción psicológica planificada” (González Bermejo, 1985, p. 109).
Varias fuentes militares han admitido que el Penal buscaba "destruir la salud mental de los presos", mientras que el psiquiatra Martín Gutiérrez, colaborador de Brito en el Penal, reconoció que "día tras día, reglamento tras reglamento, el objetivo perseguido era el de hacerlos sufrir psicológicamente" (Bloche, 1987, pp. 6-8). En el cumplimiento de este objetivo, Dolcey Brito, a diferencia de Amílcar Lobo, actuaba libremente y desempeñaba un rol más directivo que subordinado en la estructura jerárquica en la que se insertaba. Se le ha descrito como "uno de los arquitectos del monstruoso programa de experimentación psicológica del Penal", como "el cerebro detrás de un esquema científico pensado para arrasar sistemáticamente las personalidades", como conductor y ejecutor de un "desmantelamiento personal, individuo por individuo" (Uruguai, 1989, p. 222). Ajustándose a la singularidad de cada caso, Dolcey Brito no seguía siempre la misma estrategia, sino que usaba todos los medios a su alcance para lograr su objetivo, entre ellos la desinformación y la distorsión de informaciones, el ocultamiento de cartas de familiares, el aislamiento e incomunicación de los presos, desplazamientos y combinaciones selectivas de los ocupantes de las celdas, y administración de sustancias psicoactivas como flufenazina y meprobamato.

Así como el psicólogo uruguayo Dolcey Brito pensó el Penal de Libertad como una gran sala de tortura para los presos políticos, el psicólogo chileno Hernán Tuane Escaff (nacido en 1927) parece haberse obstinado en concebir su país, tras el golpe de 1973, como un gigantesco espacio dedicado, en cierto modo, a torturar al conjunto de la población (Mella, 2013). Quizás tal aserción deje de juzgarse exagerada cuando se considere la estrategia de guerra psicológica planeada por Tuane mientras estaba al frente de la Dirección de Relaciones Humanas de la Secretaria General de Gobierno del dictador Augusto Pinochet. Además de intentarse legitimar la dictadura, desacreditar a los opositores y "destruir la imagen del marxismo", había un propósito explícito de amedrentar, fragilizar, vulnerar, desestructurar, enfermar e incluso lastimar a la población al suscitar y "manejar los sentimientos traumáticos de angustia, neurosis, tragedia, inseguridad, peligro y miedo", y, de manera más precisa, "actualizando factores neurotizantes" y haciendo emerger "contenidos psicológicos latentes de índole angustiosa" y emociones como el "temor instintivo" de los delincuentes ante la perspectiva de "castigos angustiosos severos" (Dirección de Relaciones Humanas, 1974, en Baltazar 
Mozqueda, 2017, pp. 127-130). La intervención del conocimiento psicológico en esta estrategia colectiva es prácticamente la misma que encontramos en algunas torturas individuales. Quizás la estrategia de Tuane reproduzca de alguna manera su experiencia más convencional cuando auxiliaba torturas y administraba el suero de la verdad, el pentotal, a torturados por el Servicio de Investigaciones (Oliva García, 2013). El caso es que tenemos a un psicólogo torturador que además diseñó una estrategia de guerra psicológica en la que vemos reaparecer elementos que nos hacen recordar la utilización de la Psicología en la tortura.

\section{Consideraciones finales}

Una táctica reveladora de Tuane y de su equipo, en la que alcanzamos a entrever la función destructiva de la Psicología en la tortura, es aquella por la que se buscaba "desconcientizar" y así preparar el terreno para después "concientizar" en una dirección diferente (Dirección de Relaciones Humanas, 1974, en Baltazar Mozqueda, 2017, p. 129). Lo que se intentaba, en otras palabras, era desintegrar y destruir la conciencia compatible con el socialismo, aquella por la que Salvador Allende llegó al poder, para después reconstruir aquella forma de in-conciencia que se requería en el capitalismo neoliberal pinochetista. Nos acercamos así, una vez más, como en el caso de Roquet, a la técnica de Cameron consistente en la reorganización y reconstrucción del psiquismo sobre la base de su previa desorganización y destrucción.

La guerra psicológica de Tuane, como la tortura psicológica de Roquet y de Cameron, servía para destruir de algún modo el psiquismo: para desintegrar el soporte psíquico del socialismo como un requisito indispensable para poder posteriormente constituir el soporte psíquico neoliberal. ¿Cómo no ver aquí el vínculo que Naomi Klein establece entre la estrategia destructiva-reconstructiva de la conducción psíquica de Cameron y la misma estrategia destructiva-reconstructiva utilizada para la implantación del neoliberalismo en Chile a través del golpe de estado en el que Tuane tuvo un rol decisivo? Quizás Tuane sea una suerte de eslabón perdido entre los dos "doctores Shock" a los que se refiere Klein (2014): entre Milton Friedman y Ewen Cameron, entre la Escuela de Chicago y la de McGill, entre la técnica golpista y la de electrochoques, entre la dictadura y el empleo de la Psicología en la tortura (pp. 49-108). Es como si en Tuane viéramos cerrarse las dos mordazas, la psicológica y la política, de aquella pinza del imperialismo estadounidense que no deja de oprimir y lastimar a los pueblos latinoamericanos.

Considerando lo dudosa que resulta la reconstrucción en una ecuación destructiva-reconstructiva en la que solo hemos visto evidenciarse históricamente la destrucción, quizás pueda considerarse que lo único distintivo de Cameron, de Tuane y quizás también de Roquet, en contraste con Brito, Lobo y los demás, es el apéndice ideológico de un fin positivo, reconstructivo, con el que pretende justificarse el medio, así como también, tal vez, disimular el verdadero fin. ¿Y cuál es el verdadero fin? Quizás precisamente aquello que se hace pasar por medio. ¿Acaso no es lógico y comprensible que el poder busque en última instancia destruir aquello que se le contrapone? Y esto contrapuesto al poder puede ser paradójicamente el psiquismo: el mismo psiquismo creado y recreado por el poder. Si lo más común es que el mundo interno sea un lugar para ejercer el poder, aquí aparece como un reducto de resistencia contra el interrogatorio y contra cualquier otra forma de ejercicio del poder. La tortura buscaría entonces la supresión de tal reducto, la eliminación de una bolsa de resistencia, es decir, en definitiva, la sujeción del torturado, su obediencia ante el torturador, su dominación por quienes emplean al torturador, su capitulación ante el poder, su resignación a la condición de subyugado, sometido, avasallado, oprimido.

La tortura funcionaría como el golpe de estado: como un choque traumático para poner al dominado en su lugar de una vez por todas. La Psicología es aquí decisiva. Y, como hemos visto, no se necesita un psicólogo para aplicarla. De hecho, como lo demuestran los hechos, ni siquiera se requiere de un profesional de la salud mental. i̇Acaso hay que estudiar mucho para comprender las virtudes inherentes al shock del que habla Naomi Klein? Existe un testimonio en el que un torturador brasileño, un simple oficial de policía, explicaba que los torturados eran "como perros de Pavlov": el choque al principio debía "ser de alto voltaje" y luego podían aplicarse "choques más pequeños", pues la "memoria sería del choque de alto voltaje” (Murat, 2013, párr. 28). No hay que ser un psicólogo para entender esto. O mejor dicho: esto puede ser comprendido por casi todos en nuestra sociedad, pues casi todos somos un poco psicólogos, entre ellos los torturadores, los represores, los dictadores, los políticos y los economistas.

Los sujetos van convirtiéndose en psicólogos mientras que aquello que les rodea obedece cada 
vez más a funciones y determinaciones psicológicas. El mundo social ha ido psicologizándose así en el transcurso del siglo XX, y su psicologización, por cierto, implica una despolitización de lo que se vuelve psicológico, es decir, aparentemente privado, personal e íntimo (De Vos, 2012). Desde luego que esta despolitización es política en sí misma y persigue un objetivo político preciso. Como bien lo ha señalado Rancière (1998), el “más viejo trabajo del arte político" es precisamente la "despolitización" (p. 47). Despolitizar continúa siendo una de las más importantes estrategias políticas para dominar. De ahí que podamos pensar incluso, como Bourdieu (2001), en una "política de la despolitización" (99-102). Lo importante aquí es la manera en que semejante política se ha servido cada vez más de la Psicología, no solo puntualmente para torturar a quienes resisten a la despolitización, sino también, de manera constante y general, para conseguir la despolitización de los demás a través de la psicologización masiva de la sociedad. Esta orientación psicologizadora y despolitizadora parece haber cumplido un papel central y fundamental en diversos contextos en los que se ha torturado, como lo ha mostrado Coimbra (1995) en el caso brasileño. Es como si las tiranías del último siglo fueran también despotismos de la Psicología. Entendemos que esta misma Psicología no pudiera faltar en el instrumental de los torturadores nazis y franquistas en Europa, franceses en Argelia, estadounidenses en todo el mundo y latinoamericanos en regímenes dictatoriales.

\section{Referências}

Arrigo, J. M., \& Long, J. (2008). A lesson for world psychology: Denunciation and accommodation of abusive interrogations by the American Psychological Association. Preventing Torture within the Fight Against Terrorism Newsletter, 2(5), 2-7.

Baltazar Mozqueda, G. (2017). Psicología y dictadura: una aproximación a la guerra psicológica en Chile (19711989). Tesis de Licenciatura, Universidad Michoacana de San Nicolás de Hidalgo, Morelia.

Bendfeldt-Zachrisson, F. (1988). Torture as intensive repression in Latin America: the psychology of its methods and practice. International Journal of Health Services, 18(2), 301-310. Https://doi.org/10.2190/M4R4-E6WX-TKH8-NPNL

Bloche, M. G. (1987). Uruguay's military physicians: cogs in a system of state terror. Washington, D. C.: American Association for the Advancement of Science.

Bourdieu, P. (2001). Contra la política de despolitización. Ecología Política 21, 99-102.

Brasil. Comissão Nacional da Verdade (2014). Tortura. In Comissão Nacional da Verdade. Relatório (Vol. 1, pp. 327-398). Recuperado de http://www.cnv.gov.br/images/documentos/Capitulo9/Capitulo\%209.pdf

Braunstein, N., Pasternac, M., Benedito, G., \& Saal, F. (2006). Psicología: ideología y ciencia. Ciudad de México: Siglo XXI.

Campbell, T. A. (2007). Psychological assessment, diagnosis, and treatment of torture survivors: a review. Clinical Psychology Review, 27(5), 628-641. https://doi.org/10.1016/j.cpr.2007.02.003

Carpintero, E., \& Vainer, A. (2005). Las huellas de la memoria: psicoanálisis y salud mental en la Argentina de los 60' y 70'. Buenos Aires: Topía.

Carvalho, P. (Ed.) (2013). A verdade é revolucionária: testemunhos e memória de psicólogas e psicólogos sobre a ditadura civil-militar brasileira (1964-1985). Brasilia, D. F: Conselho Federal de Psicología.

Central Inteligence Agency - CIA. (1963). Kubark counterintelligence Interrogation manual. 1963. Recuperado de http://nsarchive.gwu.edu/NSAEBB/NSAEBB27/01-04.htm

Coimbra, C. M. B. (1995). Guardiães da ordem: algumas práticas PSI no Brasil do “milagre”. Rio de Janeiro, RJ: Oficina do Autor.

Cohn, G., Thompson, G., \& Matthews, M. (1997, 27 de enero). Torture was taught by CIA; declassified manual details the methods used in Honduras; Agency denials refuted. The Baltimore Sun. Recuperado de http://www.hartford-hwp.com/archives/40/055.html

Costanzo, M., Gerrity, E., \& Lykes, M. B. (2007). Psychologists and the use of torture in interrogations. Analyses of Social Issues and Public Policy, 7(1), 7-20. https://doi.org/10.1111/j.1530-2415.2007.00118.x

Czech, H. (2014). Abusive medical practices on "euthanasia" victims in Austria during and after World War II. In S. Rubenfeld, \& S. Benedict (Eds), Human subjects research after the Holocaust (pp. 109-125). New York, NY: Springer. 
Dawson, A. S. (2015). Salvador Roquet, María Sabina, and the Trouble with Jipis. Hispanic American Historical Review 95(1), 103-133. https://doi.org/10.1215/00182168-2836928

De Vos, J. (2012). Psychologisation in times of globalisation. Londres: Routledge.

Dobles, I. (1990). Psicología de la tortura. In B. Jiménez-Domínguez (Coord.), Aportes críticos a la psicología en Latinoamérica. Guadalajara: Universidad de Guadalajara.

Eban, K. (2007). Rorschach and Awe. Vanity Fair. Recuperado de http:/ /www.vanityfair.com/news/2007/07/torture200707

Fanon, F. (1965). A dying colonialism. New York, NY: Grove Press.

Fiske, S. T., Harris, L. T., \& Cuddy, A. J. (2004). Why ordinary people torture enemy prisoners. Science, 306(5701), 1482-1483. https://doi.org/10.1126/science.1103788

Friedlander, H. (1995). The origins of Nazi genocide: from euthanasia to the final solution. Chapel Hill, NC: University of North Carolina Press.

González-Bermejo, E. (1979). Rehacer al hombre: tortura y exilio. Nueva Sociedad 44, 107-115.

González Bermejo, E. (1985). Las manos en el fuego. Montevideo: Banda Oriental.

Gorman, W. (2001). Refugee survivors of torture: trauma and treatment. Professional Psychology: Research and Practice, 32(5), 443-451. https://doi.org/1037/0735-7028.32.5.443

Haney, C., Banks, W. C., \& Zimbardo, P. G. (1973). A study of prisoners and guards in a simulated prison. Naval Research Reviews, 9(1-17). Recuperado de http://www.zimbardo.com/downloads/1973\%20A\%20Study\%20of\%20Prisoners\%20and\%20Guards,\%20Naval\%20Research\%20Reviews.pdf

Hanscom, K. L. (2001). Treating survivors of war trauma and torture. The American Psychologist, 56(11), 1032-1039.

Jahn, G. (2012, 9 May). Vienna buries last remains of victims of Nazi medical mistreatment. National Post. Recuperado de http://news.nationalpost.com/news/world/vienna-buries-last-remains-of-victims-of-nazi-medical-mistreatment

Janoff-Bulman, R. (2007). Erroneous assumptions: popular belief in the effectiveness of torture interrogation. Peace and Conflict: Journal of Peace Psychology, 13(4), 429-435. https://ddoi.org/10.1080/10781910701665766

Ketzer, E., \& Crescenzi, A. (2002). Addressing the Psychosocial and Mental Health Needs of Tibetan Refugees in India. In Joop de Jong (Ed), Trauma, war, and violence: public mental health in socio-cultural context (pp. 283-316). New York, NY: Kluwer.

Klein, N. (2014). La doctrina del shock: el auge del capitalismo del desastre. Ciudad de México: Paidós.

Le Cudennec, J. (2009). David Galula et Roger Trinquier: perceptions croisées de la contre-insurrection. TTU online. Recuperado de http:/ / www.ttu.fr/david-galula-et-roger-trinquier-perceptions-croisees-de-la-contre-insurrection/

Lindén, M., Björklund, F., \& Bäckström, M. (2016). What makes authoritarian and socially dominant people more positive to using torture in the war on terrorism? Personality and Individual Differences 91, 98-101. https://doi.org/10.1016/j.paid.2015.11.058

Lira, E. (2008). Psicología, ética y seguridad nacional: el rol de los psicólogos. Psykhe, 17(2), 5-16. Recuperado de http://www.scielo.cl/pdf/psykhe/v17n2/art01.pdf

Lira, E., \&Weinstein, E. (1984). Psicoterapia y represión política. Ciudad de México: Siglo Veintiuno.

Lobo, A. (1998). La hora del lobo, la hora del carnero. Córdoba: EDELP.

López de la Torre, C. F. (2013). Miguel Nazar Haro y la guerra sucia en México. Grafía 10(1), 56-72. Recuperado de http://revistas.fuac.edu.co/index.php/grafia/article/view/350/333

McCoy, A. W. (2006). A question of torture. CIA interrogation, from the Cold War to the war on terror. New York, NY: Metropolitan.

McCoy, A. W. (2009, 8 de junio). Confronting the CIA's Mind Maze:. America's political paralysis over torture. Antiwar.com. Recuperado de http://www.truth-out.org/archive/item/84471:confronting-the-cias-mind-maze-americas-political-paralysis-over-torture

Magalhães, v. S. (2009). Entrevista com Vera Sílvia Magalhães. Câmara dos Deuputados. Recuperado de http:// www2.camara.leg.br/a-camara/conheca/historia/historia/historiaoral/Memoria\%20Politica/Depoimentos/ vera-silvia-magalhaes/texto 
Marín, N. (1985, 28 de marzo). Desde 62 el Maoísmo empezó a actuar con obreros y campesinos: Emery Ulloa. Excélsior. Recuperado de http://arorci.blogspot.mx/2013/03/desde-62-el-maoismo-empezo-actuar-con.html

Marín, N. (1985, 29 de marzo). Desde 62 el Maoísmo empezó a actuar con obreros y campesinos. Segunda parte de cuatro. Excélsior. Recuperado de http://arorci.blogspot.mx/2013/03/desde-62-el-maoismo-empezo-actuar-con.html

Martens, D. (2004). Unfit to live. Canadian Medical Association Journal, 171(6), 619-620. https://doi.org/10.1503/ cmaj.1041335

Mella, C. (2013, 16 de septiembre). La mente del horror: psicología al servicio de la dictadura. Clarín. Recuperado de http://www.elclarin.cl/web/noticias/politica/9190-la-mente-del-horror-psicologia-al-servicio-de-la-dictadura.html

Milgram, S. (1974). Obedience to authority: an experimental view. New York, NY: Harper \& Row.

Monge, R. (2002, 3 de agosto). Nazar Haro y la psicotortura. Processo. Com. Mx. Recuperado de http://www.proceso.com.mx/187992/nazar-haro-y-la-psicotortura

Murat, L. (2013). El testimonio de Lúcia Murat. Programa Ibermedia. Recuperado de http://www.programaibermedia.com/nuestras-cronicas/lucia-murat-a-la-comision-de-la-verdad-de-rio/

Oliva García, J. (2013). Los torturadores: quiénes son y dónde están. Lo que no dice el Informe Valech. Tercera Información. Recuperado de http://www.tercerainformacion.es/antigua/spip.php?article52824

Parker, I. (2010). La psicología como ideología. Madrid: Catarata.

Patel, N., Williams, A. C. D. C., \& Kellezi, B. (2016). Reviewing outcomes of psychological interventions with torture survivors: conceptual, methodological and ethical issues. Torture, 26(1), 2-16.

Pope, K. S., \& Garcia-Peltoniemi, R. E. (1991). Responding to victims of torture: clinical issues, professional responsibilities, and useful resources. Professional Psychology: Research and Practice, 22(4), 269-276.

Qouta, S., \& El-Sarraj, E. (2002). Community mental health as practiced by the Gaza Community Mental Health Program. In Joop de Jong (Ed), Trauma, war, and violence: public mental health in socio-cultural context (pp. 317-336). New York, NY: Kluwer.

Ramírez, I. (1985, 26 de agosto). El creador de la Psicosíntesis, terapia con hongos, también engañó a Sabi. Proceso. Recuperado el 5 junio de 2017 de http://www.proceso.com.mx/284662/el-creador-de-la-psicosintesis-terapia-con-hongos-tambien-engano-a-sabi

Rancière, J. (1998). Aux bords du politique. París: Gallimard.

Riquelme, H. (2004). Ética profesional en tiempos de crisis: médicos y psicólogos en las dictaduras de América del Sur. PoliS: Revista Latinoamericana 8. Recuperado de http://polis.revues.org/6129

Robin, M.-M. (2003). Escadrons de la mort, l'école française. París: La Découverte.

Rodríguez Castañeda, R. (2013). El policía; perseguía, torturaba, mataba. Ciudad de México: Grijalbo.

Romeu, I. E. (2011, 11 de junio). A torturada fala com o médico da tortura. Revista O Viés? Recuperado de http:// www.revistaovies.com/extras/2011/06/a-torturada-fala-com-o-medico-da-tortura/

Roquet, S. (1971, 15 de noviembre). Entrevista a Salvador Roquet: primera parte. Revista Piedra Rodante. Recuperado de http://joechipnotdie.blogspot.mx/2009/03/entrevista-salvador-roquet-primera.html

Rose, N. (1989). Governing the soul: the shaping of the private self. London: Free Association Books.

Rose, N. (1998). Inventing our selves: psychology, power and personhood. Cambridge: Cambridge University Press.

Saldarriaga, G. (2009). Psicólogos y tortura: notas sobre la batalla por el futuro de la psicología. Revista de Psicología Universidad de Antioquia, 1(2), 61-82. Recuperado de http://aprendeenlinea.udea.edu.co/revistas/index.php/ psicologia/article/view/10021/9238

Sánchez, R. (2003, 9 de noviembre). Me torturaron con drogas naturales y químicas: Ulloa. Crónica. Recuperado de http://www.cronica.com.mx/notas/2003/93521.html

Scott, P. D. (2014). American war machine: deep politics, the CIA global drug connection, and the road to Afghanistan. Lanham, MD: Rowman \& Littlefield.

Seligman, M. E. P. (1975). Helplessness: on depression, development, and death. San Francisco, CA: W. H. Freeman. 
Senate Select Committee on Intelligence (2014). Committee Study of the Central Intelligence Agency's Detention and Interrogation Program. Findings and Conclusions. Recuperado de https://web.archive.org/web/20141209165504/ http://www.intelligence.senate.gov/study2014/sscistudyl.pdf

Soldz, S. (2008). Healers or interrogators: psychology and the United States torture regime. Psychoanalytic Dialogues, 18(5), 592-613. https://doi.org/10.1080/10481880802297624

Soldz, S. (2011). Fighting torture and psychologist complicity. Peace Review: A Journal of Social Justice, 23(1), 12-20. https://doi.org/10.1080/10402659.2011.548240

Staub, E. (1990). The psychology and culture of torture and torturers. In P. Suedfeld (Ed.), Psychology and torture (pp. 49-76). Washington, D. C.: Hemisphere Publishing

Suedfeld, P. (1990). Psychology and torture. New York, NY: Hemisphere.

Suedfeld, P. (2007). Torture, interrogation, security, and psychology: absolutistic versus complex thinking. Analyses of Social Issues and Public Policy 7(1), 55-63. https://doi.org/10.1111/j.1530-2415.2007.00125.x

Sullivan, A. (2009, 21 de abril). The case of Richard Wilhelm Hermann Bruns, et al. The Atlantic. Recuperado de https://www.theatlantic.com/daily-dish/archive/2009/04/the-case-of-richard-wilhelm-hermann-bruns-e$\mathrm{t}-\mathrm{al} / 202868 /$

Suprema Corte de Noruega (1946). Trial of Kriminalsekretar: Richard Wilhelm Hermann Bruns and two others. Recuperado de http://www.worldcourts.com/ildc/eng/decisions/1946.07.03_Norway_v_Bruns.pdf

Torres, J. (2008). Nazar, la historia secreta: el hombre detrás de la guerra sucia. Ciudad de México: Debate.

Uruguai. Servio de Paz y Justicia - SERPAJ. (1989). Uruguay nunca más: informe sobre la violación de los derechos humanos (1972-1985). Montevideo: o autor. Recuperado de http://www.memoriaenelmercosur.educ.ar/ wp-content/uploads/2009/05/completo.pdf

Vallejo Nájera, A. (1939). La locura en la guerra: psicopatología de la guerra española. Valladolid: Santarén.

Van Ommeren, M., Sharma, B., Prasain, D., \& Poudyal, B. N. (2002). Addressing human rights violations: a public mental health perspective on helping torture survivors in Nepal. In Joop de Jong (Ed.), Trauma, war, and violence: public mental health in socio-cultural context (pp. 259-281). New York, NY: Kluwer.

Vargas Llosa, M. (1990, 1 de septiembre). México es la dictadura perfecta. El País. Recuperado de http://elpais. com/diario/1990/09/01/cultura/652140001_850215.html

Viki, G. T., Osgood, D., \& Phillips, S. (2013). Dehumanization and self-reported proclivity to torture prisoners of war. Journal of Experimental Social Psychology, 49(3), 325-328. https://doi.org/10.1016/j.jesp.2012.11.006

Vinyes Ribas, R. (2001). Construyendo a Caín: diagnosis y terapia del disidente: las investigaciones psiquiátricas militares de Antonio Vallejo Nágera con presas y presos políticos. Ayer (44), 227-250.

Welch, B. L. (2010). The torturer's apprentice: psychology and 'enhanced interrogations'. Global Dialogue, $12(1), 1-14$.

Wilson, J. P., \& Drozdek, B. (Eds) (2004). Broken spirits: the treatment of traumatized asylum seekers, refugees and war and torture victims. Londres: Routledge.

Windrem, R. (2014). CIA paid torture teachers more than $\$ 80$ million. NBC News. Recuperado de http://www.nbcnews.com/storyline/cia-torture-report/cia-paid-torture-teachers-more-80-million-n264756

Wolfson, P. E. (2014). Psychedelic experiential pharmacology: pioneering clinical explorations with Salvador Roquet (How i came to all of this: ketamine, admixtures and adjuvants, Don Juan and Carlos Castaneda too): an interview with Richard Yensen. International Journal of Transpersonal Studies, 33(2), 160-174. Recuperado de http://digitalcommons.ciis.edu/cgi/viewcontent.cgi?article=1026\&context=ijts-transpersonalstudies

\section{David Pavón-Cuéllar}

Doutor em Psicologia - Universidad de Santiago de Compostela e Doutor em Filosofia - Université de Rouen.

E-mail: davidpavoncuellar@gmail.com 
Endereço para envio de correspondência:

David Pavón-Cuéllar, Facultad de Psicología de la UMSNH, Francisco Villa 450, Dr. Miguel Silva González, 58110 Morelia, Michoacán, México

Recebido 30/06/2017

Reformulação 14/09/2017

Aprovado 20/09/2017

Received 06/30/2017

Reformulated $09 / 14 / 2017$

Approved 09/20/2017

Recebido 30/06/2017

Reformulado 14/09/2017

Aceptado 20/09/2017

Cómo citar: Pavón-Cuéllar, David. (2017). Psicología y destrucción del psiquismo: la utilización profesional del conocimiento psicológico para la tortura de presos políticos. Psicologia: Ciência e Profissão, 37(n. spe), 11-27. https://doi.org/10.1590/1982-3703010002017

Como citar: Pavón-Cuéllar, David. (2017). Psicologia e destruição do psiquismo: a utilização profissional do conhecimento psicológico para a tortura de presos políticos. Psicologia: Ciência e Profissão, 37(n. spe), 11-27. https://doi.org/10.1590/1982-3703010002017

How to cite: Pavón-Cuéllar, David. (2017). Psychology and destruction of the psyche: the professional use of psychological knowledge for torture of political prisoners. Psicologia: Ciência e Profissão, 37(n. spe), 11-27. https://doi.org/10.1590/1982-3703010002017 